\title{
Pathophysiology of Cerebral Ischemia: Role of Oxidative/Nitrosative Stress
}

\author{
Hiba A. Awooda ${ }^{1,2}$ \\ ${ }^{1}$ College of Applied Sciences, AlMaarefa University, Riyadh, Saudi Arabia \\ ${ }^{2}$ Faculty of Medicine, Al Neelain University, Khartoum, Sudan \\ Email: h_alsheekh82@yahoo.com
}

How to cite this paper: Awooda, H.A (2019) Pathophysiology of Cerebral Ischemia: Role of Oxidative/Nitrosative Stress. Journal of Biosciences and Medicines, 7, 20-28.

https://doi.org/10.4236/jbm.2019.73003

Received: May 29, 2018

Accepted: March 3, 2019

Published: March 6, 2019

Copyright (c) 2019 by author(s) and Scientific Research Publishing Inc. This work is licensed under the Creative Commons Attribution International License (CC BY 4.0).

http://creativecommons.org/licenses/by/4.0/

\begin{abstract}
Stroke is a devastating disease with a complex pathophysiology; it ranks second to ischemic heart disease as a cause of death and long-term disability. Tissue damage results from diverse mechanisms with central involvement of free radicals' overproduction that results in oxidative stress and hence contributes to brain damage. Free radicals [Reactive oxygen species/Reactive nitrogen species] play central a role in the diverse normal physiological processes and as defense mechanisms against harmful substances. When the rate of their production exceeds the antioxidant capacity of the body, oxidative stress occurs. Oxidative stress is implicated in the pathogenesis of various diseases including hypertension, atherosclerosis, diabetes mellitus and cancer; they mediate damage to cell structures, lipid peroxidation, protein denaturation, nucleic acid and DNA damage.
\end{abstract}

\section{Keywords}

Reactive Oxygen Species, Reactive Nitrogen Species, Cerebral Ischemia, Oxidative Stress

\section{Introduction}

Stroke is the second leading cause of death and the third leading reason of permanent disability in adults worldwide [1] [2]. The predominance affected group is over the age of 65 years, but all ages are at a risk of having a stroke even children and infants [3]. According to the World Health Organization (WHO), stroke has globally affected a total of 15 million people each year. Five million suffer from permanent disability, and 5.5 million people accede to varying levels of stroke-related disabilities [4]. The prevalence rate of stroke is assessed to be about 400 - 800 per 100,000 persons [5]. Incidence rates for stroke ranged from 
41 per 100,000 population per year in Nigeria (1971-74) to 316/100,000 per year in urban Dares-Salaam (Tanzania) [6]. For native Indians, the overall age-adjusted prevalence rate for stroke is between about 84 - 262 per 100,000 persons in rural areas and between about 334 - 424 per 100,000 persons in urban areas [7] with an average prevalence rate of $90-222$ per 100,000 persons.

The WHO describes a stroke as an interruption to the supply of blood to the brain, with "rapidly developing clinical signs of focal or global disruption of cerebral function, with signs lasting 24 hours or longer, or leading to death with no apparent cause other than vascular origin". There are two major mechanisms causing brain damage in stroke patients: Hemorrhage and Ischemia which represents about $70 \%$ to $80 \%$ of all strokes' cases. It has been reported that up to $30 \%$ of all ischemic strokes will ultimately undergo hemorrhagic transformation [8]. More than any other organ of the body, the brain integrity highly depends on the continuous blood supply of oxygen and glucose for covering its energy demands. Thus, low respiratory reserve and complete dependence on aerobic metabolism make brain tissue particularly vulnerable to effects of ischemia [3]. The pathophysiology of ischemic stroke is complex and recent molecular, cellular, and animal models and postmortem brain studies have revealed that multiple cellular changes have been implicated, including oxidative stress/mitochondrial dysfunction, inflammatory responses, micro RNA alterations, and marked changes in brain proteins [1]. Recent researches have suggested link between an excessive generation of reactive oxygen species [ROS] and the development of neuronal death in acute brain injury like brain trauma and cerebral ischemia [9]. Excessive ROS generation can induce the functional and structural damage of neuronal cells and may play an important role in the pathophysiology of cerebral ischemia [10] [11].

\section{Mechanisms of Ischemic Brain Injury}

Cerebral ischemia results in a number of hemodynamic, biochemical, and neurophysiologic alterations that can be linked clinically to behavioral and pathologic disturbances. The pathophysiological mechanisms of stroke are complex, and the ischemic cascade is rapidly initiated which comprises a series of subsequent biochemical events that may be neuroprotective or detrimental to the brain [12]. The ischemic incident begins with reduced blood flow to the areas supplied by the obstructed arteries. Neuronal tissues are mainly affected by depletion of high-energy phosphate compound adenosine triphosphate (ATP). Consequently, in cerebral ischemic cells no ATP produced due to hypoxia and lack of glucose supply hence maintenance of $\mathrm{Ca}^{2+}$ homeostasis is lost due to insufficient ATP to fuel extrusion pumps, while the resting membrane potential is also disrupted due to dysfunction of the $\mathrm{Na}^{+} / \mathrm{K}^{+}$ATPase pumps, leading to "anoxic depolarisation" [13]. The resulting ionic imbalance within the neuronal and glial cells manifests in the development of tissue acidosis, cytotoxic edema and ultimately cell necrosis [3]. The cellular events leading to ischemic neuronal 
death have been widely studied in animal models of stroke [14] [15] and the probable sequence of events involved in ischemic neuronal death is demonstrated in Figure 1 which emphasis the detrimental effects of oxidative/nitrosative stress in cerebral ischemia.

\section{Role of Reactive Oxygen Species in the Normal Brain Physiology}

Hence all cells use oxygen to produce energy; free radicals are formed as a result of ATP production by the mitochondria and known as reactive oxygen species (ROS). ROS play a dual role as toxic and beneficial substances. At low or moderate concentration, ROS had beneficial effects while at high concentrations, they result in oxidative stress, a harmful process that can damage the cellular structure [16].

In the nervous system ROS are mainly produce by microglia and astrocytes. They play important role in regulation of neuronal signaling. Periphery; ROS result in ATP inhibiting acetylcholine release from motor nerve endings, while centrally may enhance or depress synaptic transmission in the central nervous system. In the hippocampus, ROS promote protein kinase C-dependent; hence increases the excitatory postsynaptic potentials [17]. Moreover; ROS can induce synaptic long term potentiation (LTP) that required for memory formation [18].

\section{Oxidative/Nitrosative Stress in Cerebral Ischemia}

Oxidative/Nitrosative stress generally describes as a condition in which cellular

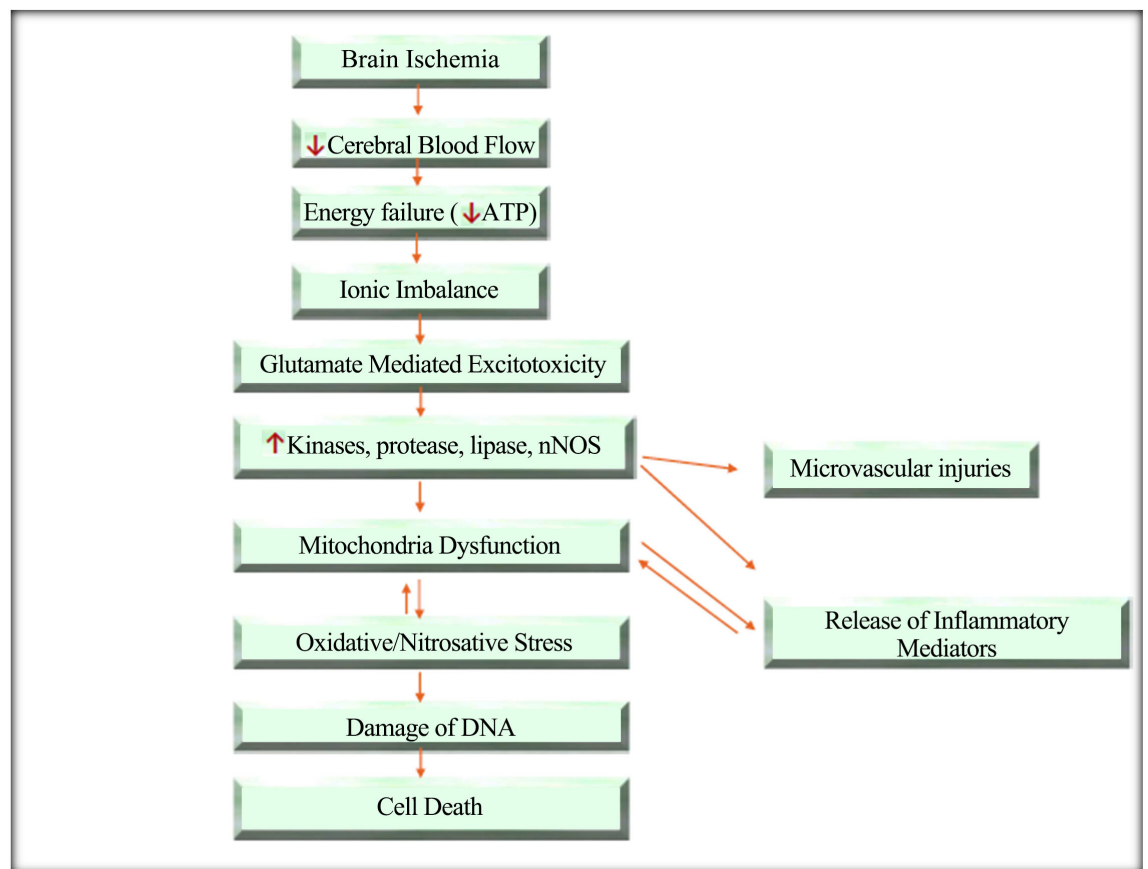

Figure 1. Major processes and mediators involved in the pathophysiology of focal cerebral ischemia reperfusion, excitotoxicity, ionic imbalance, oxidative and apoptotic mechanisms. 
antioxidant defenses are inadequate to completely inactivate the reactive oxygen species (ROS) and reactive nitrogen species (RNS) generated because of excessive production of ROS/RNS, loss of antioxidant defenses, or both [19]. A major consequence of oxidative/nitrosative stress is damage to nucleic acid bases, lipids, and proteins, which can severely compromise cell health and viability or induce a variety of cellular responses through generation of secondary reactive species, ultimately leading to cell death by necrosis or apoptosis [20].

Oxidative stress leading to ischemic cell death involves the formation of ROS including superoxide anions $\left(\mathrm{O}_{2}^{-}\right)$, hydroxyl radicals $\left(\mathrm{OH}^{-}\right)$, hypochlorous acid ( $\mathrm{HOCl})$, hydrogen peroxide $\left(\mathrm{H}_{2} \mathrm{O}_{2}\right)$, and nitric oxide-derived peroxynitrite (NO and $\mathrm{ONOO}^{-}$). During oxidative stress, rapid overproduction of free radicals overwhelms the detoxification and scavenging capacity of cellular antioxidant enzymes like superoxide dismutase (SOD); catalase (CAT); glutathione peroxidase (GPx) and non-enzymatic antioxidants like vitamin E, vitamin $\mathrm{C}$ and glutathion resulting in a severe and immediate damage to cellular proteins, DNA and lipids [21].

Following cerebral ischemia/reperfusion there is increased production of ROS/RNS which one of the major causes of neuronal injury. Particularly, the brain is highly susceptible to free radical mediated insult owing to its high lipid content [21]. ROS levels are elevated in the cerebral vasculature during reperfusion period [22], and suspected to be an underlying cause of post-ischemic endothelial dysfunction, however, their enzymatic source(s) is yet to be defined [11]. The (nicotinamide adenine dinucleotide phosphate) NADPH oxidases are the only enzymes yet discovered with the primary function of generating superoxide and ROS in the cerebral vasculature under physiological conditions [23].

Oxygen radicals are also produced during enzymatic conversions, such as the cyclooxygenase-dependent conversion of arachidonic acid to prostanoids and the degradation of hypoxanthine, especially upon reperfusion. Furthermore, free radicals are also generated during the inflammatory response after ischemia. Moreover, oxidation of xanthine and hypoxanthine by xanthine oxidase is accompanied with generation of $\mathrm{O}_{2}^{-}$and $\mathrm{H}_{2} \mathrm{O}_{2}$ that contributes to neuronal injury during reperfusion [24].

Oxidative/nitrosative stresses are modulated by enzyme systems such as SOD and the nitric oxide synthase (NOS) family [25]. Mice with enhanced expression of SOD show reduced injury after cerebral ischemia, whereas those with a deficiency show increased injury, proving that excessive oxygen radical production is fundamental to ischemic brain injury, while in case of NOS activation during ischemia, superoxide reacts avidly with NO leading to decrease NO bioavailability and the formation of peroxynitrite a highly reactive and damaging species that causes nitration of tyrosine residues on proteins [11] [26].

ROS/RNS provoke inflammatory responses resulting in the release of proinflammatory cytokines and chemokines. Moreover, it stimulates leukocyte adhesion molecule and cytokine gene expression via activation of transcription fac- 
tors such as Nuclear Factor Kappa- $\beta$ (NF- $\kappa \beta)$. Thus ROS/RNS not only cause direct cell injury but also increase leukocyte activation, chemotaxis, and leukocyte—endothelial adherence after reperfusion [27] [28].

\section{Consequence of Oxidative/Nitrosative Stress in Cerebral Ischemia}

ROS/NOS result in significant cellular destructive effects, that eventually resulting in tissue destruction and cell death; these effects include: lipid peroxidation, protein denaturation, nucleic acid and DNA damage, damage to the cytoskeletal structure, and chemotaxis [25].

\section{Lipid Peroxidation and Malondialdehyde}

Lipid peroxidation is one of the major consequences of ROS/NOS mediated injury to the brain. It produces structural and functional damage to membranes as well as several secondary products [29].

Oxidative stress induced peroxidation of membrane lipids can be very damaging because it leads to alterations in the biological properties of the membrane, such as the degree of fluidity, and can lead to inactivation of membrane-bound receptors or enzymes, which in turn may impair normal cellular function and increase tissue permeability. Moreover, lipid peroxidation may contribute to and amplify cellular damage resulting from generation of oxidized products, some of which are chemically reactive and covalently modify critical macromolecules [29].

There are many toxic aldehydes generated during lipid peroxidation: $\alpha, \beta$ unsaturated reactive aldehydes, such as malondialdehyde (MDA), 4-hydroxy-2nonenal (HNE), and 2-propenal (acrolein), and isoprostaness, 4-hydroxynonenal (4-HNE), which damages a variety of ion channels, transporter, and cytoskeletal proteins. These products of lipid peroxidation have therefore commonly been used as biomarkers of oxidative stress/damage [11] [22].

Malondialdehyde (MDA) is a physiologic ketoaldehyde generated by the attack of free radicals on polyunsaturated fatty acids of cell membranes; its measurement provides information of oxidative injury in vivo. The excess MDA produced as a result of tissue injury; can combine with free amino groups of proteins producing MDA modified protein adducts. Modification of proteins by MDA could alter their biological properties [25]. Moreover, MDA modified proteins are immunogenic, and autoantibodies against MDA have been detected in the sera of rabbits and humans, some studies have reported that the titer of these autoantibodies is associated with the pathogenesis and may predict progression of atherosclerosis and myocardial infarction and strokes [19]. In addition, it has been shown to exhibit facile reactivity with various biomolecules, including proteins, DNA, and phospholipids, generating stable products at the end of a series of reactions that are thought to contribute to the pathogenesis of many diseases [30]. 
Quantification of lipid peroxidation end products is considered to be a measure of whole-body oxidative damage. MDA correlated very well with the size of ischemic stroke and clinical outcome, despite its relevant methodological limitations; MDA is one of the most frequently used indicators of lipid peroxidation [31]. Compared with free radicals, the aldehydes are relatively stable and can diffuse within or even escape from the cell and attack targets far from the site of the original event. They therefore are not only end products and remnants of lipid peroxidation processes but also may act as "second cytotoxic messengers" for the primary reactions [32].

\section{DNA Oxidation}

It has been reported that the damage of nuclear DNA during cerebral ischemia may occur with two different mechanisms: oxidative modification and endonuclease mediated DNA fragmentation. DNA degradation would take place in the late stage of cell death; oxidative DNA damage is likely to represent an early event [33]. It may activate repair enzymes leading to a rapid depletion of intracellular energy [34] [35].

Different types of oxidative DNA damage were reported such as: DNA base lesions, particularly 8-hydroxy-2-deoxy-guanosine (8-OHdG), DNA strand breaks, and DNA-protein cross-links. 8-OHdG is the most commonly studied biomarker for oxidative DNA damage due to its high specificity and relative abundance in DNA. Plasma levels of 8-OHdG were found to be increased in an animal model of ischemic stroke, with a significant association with brain content of 8-OHdG [36].

\section{Conclusion}

Free radical production is increased in ischemic and hemorrhagic stroke, leading to oxidative stress that contributes to brain damage. However, it is important to identify the functions of ROS as signaling molecules and in the normal regulation of cerebrovascular structure. A balanced between level of ROS/NOS and antioxidant system is required for normal physiological functioning and to hinder the mechanisms triggering the progression of apoptosis or necrosis following ischemia reperfusion. Further studies are needed to explore the molecular mechanisms by which antioxidants prevent the harmful effects of oxidative stress in cerebral ischemia to be implicated in management of stroke patients.

\section{Conflicts of Interest}

The author has no competing interests.

\section{References}

[1] Yesilot, N., Putaala, J., Bahar, S.Z. and Tatlisumak, T. (2017) Ethnic and Geographical Differences in Ischaemic Stroke among Young Adults. Current Vascular Pharmacology, 15, 416-429.https://doi.org/10.2174/1570161115666170202161719 
[2] Lozano, R., Naghavi, M., Foreman, K., Lim, S., Shibuya, K., Aboyans, V., et al. (2012) Global and Regional Mortality from 235 Causes of Death for 20 Age Groups in 1990 and 2010: A Systematic Analysis for the Global Burden of Disease Study 2010. The Lancet, 380, 2095-2128. https://doi.org/10.1016/S0140-6736(12)61728-0

[3] Deb, P., Sharma, S. and Hassan, K.M. (2010) Pathophysiologic Mechanisms of Acute Ischemic Stroke: An Overview with Emphasis on Therapeutic Significance beyond Thrombolysis. Pathophysiology, 17, 197-218. https://doi.org/10.1016/j.pathophys.2009.12.001

[4] WHO, WHF and WSF (2011) Global Atlas on Cardiovascular Disease Prevention and Control.

[5] Banerjee, T.K., Roy, M.K. and Bhoi, K.K. (2005) Is Stroke Increasing in India-Preventive Measures That Need to Be Implemented. Journal of the Indian Medical Association, 103, 162-166.

[6] Thrift, A.G., Cadilhac, D.A., Thayabaranathan, T., Howard, G., Howard, V.J., Rothwell, P.M., et al. (2014) Global Stroke Statistics. International Journal of Stroke, 9, 6-18. https://doi.org/10.1111/ijs.12245

[7] Gupta, R., Joshi, P., Mohan, V., Reddy, K.S. and Yusuf, S. (2008) Epidemiology and Causation of Coronary Heart Disease and Stroke in India. Heart, 94, 16-26. https://doi.org/10.1136/hrt.2007.132951

[8] Jickling, G.C., Liu, D., Stamova, B., Ander, B.P., Zhan, X., Lu, A., et al. (2014) Hemorrhagic Transformation after Ischemic Stroke in Animals and Humans. Journal of Cerebral Blood Flow \& Metabolism, 34, 185-199. https://doi.org/10.1038/jcbfm.2013.203

[9] Valko, M., Leibfritz, D., Moncol, J., Cronin, M.T.D., Mazur, M. and Telser, J. (2007) Free Radicals and Antioxidants in Normal Physiological Functions and Human Disease. International Journal of Biochemistry and Cell Biology, 39, 44-84. https://doi.org/10.1016/j.biocel.2006.07.001

[10] Niizuma, K., Endo, H. and Chan, P.H. (2009) Oxidative Stress and Mitochondrial Dysfunction as Determinants of Ischemic Neuronal Death and Survival. Journal of Neurochemistry, 109, 133-138. https://doi.org/10.1111/j.1471-4159.2009.05897.x

[11] Awooda, H.A., Lutfi, M.F. and Saeed, A.M. (2015) Oxidative/Nitrosative Stress in Rats Subjected to Focal Cerebral Ischemia/Reperfusion. International Journal of Health Sciences, 9, 17-24.

[12] Doyle, K.P., Simon, R.P. and Stenzel-Poore, M.P. (2008) Mechanisms of Ischemic Brain Damage. Neuropharmacology, 55, 310-318.

https://doi.org/10.1016/j.neuropharm.2008.01.005

[13] Jarvis, C.R., Anderson, T.R. and Andrew, R.D. (2001) Anoxic Depolarization Mediates Acute Damage Independent of Glutamate in Neocortical Brain Slices. Cerebral Cortex, 11, 249-259. https://doi.org/10.1093/cercor/11.3.249

[14] Kanekar, S.G., Zacharia, T. and Roller, R. (2012) Imaging of Stroke: Part 2, Pathophysiology at the Molecular and Cellular Levels and Corresponding Imaging Changes. American Journal of Roentgenology, 198 63-74. https://doi.org/10.2214/AJR.10.7312

[15] Khoshnam, S.E., Winlow, W., Farzaneh, M., Farbood, Y. and Moghaddam, H.F. (2017) Pathogenic Mechanisms Following Ischemic Stroke. Neurological Sciences, 38, 1167-1186. https://doi.org/10.1007/s10072-017-2938-1

[16] Halliwell, B. (2007) Biochemistry of Oxidative Stress. Biochemical Society Transactions, 35, 1147-1150.

[17] Giniatullin, A.R., Grishin, S.N., Sharifullina, E.R., Petrov, A.M., Zefirov, A.L. and 
Giniatullin, R.A. (2005) Reactive Oxygen Species Contribute to the Presynaptic Action of Extracellular ATP at the Frog Neuromuscular Junction. The Journal of Physiology, 565, 229-242. https://doi.org/10.1113/jphysiol.2005.084186

[18] Hu, D. (2006) Aging-Dependent Alterations in Synaptic Plasticity and Memory in Mice That Overexpress Extracellular Superoxide Dismutase. Journal of Neuroscience, 26, 3933-3941. https://doi.org/10.1523/JNEUROSCI.5566-05.2006

[19] Stocker, R. (2004) Role of Oxidative Modifications in Atherosclerosis. Physiological Reviews, 84, 1381-1478. https://doi.org/10.1152/physrev.00047.2003

[20] Jin, R., Yang, G. and Li, G. (2010) Inflammatory Mechanisms in Ischemic Stroke: Role of Inflammatory Cells. Journal of Leukocyte Biology, 87, 779-789. https://doi.org/10.1189/jlb.1109766

[21] Chan, P.H. (2001) Reactive Oxygen Radicals in Signaling and Damage in the Ischemic Brain. Journal of Cerebral Blood Flow and Metabolism, 21, 2-14. https://doi.org/10.1097/00004647-200101000-00002

[22] Awooda, H., Lutfi, M.F., Sharara, G.M. and Saeed, M. (2013) Role of N-Nitro-LArginine-Methylester as Anti-Oxidant in Transient Cerebral Ischemia and Reperfusion in Rats. Experimental \& Translational Stroke Medicine, 5, 1.

http://www.embase.com/search/results?subaction=viewrecord\&from=export\&id=L $\underline{368170738}$

[23] Drummond, G.R., Selemidis, S., Griendling, K.K. and Sobey, C.G. (2011) Combating Oxidative Stress in Vascular Disease: NADPH Oxidases as Therapeutic Targets. Nature Reviews Drug Discovery, 10, 453-471. https://doi.org/10.1038/nrd3403

[24] Miller, A.A., Drummond, G.R. and Sobey, C.G. (2006) Novel Isoforms of NADPHOxidase in Cerebral Vascular Control. Pharmacology and Therapeutics, 111, 928-948. https://doi.org/10.1016/j.pharmthera.2006.02.005

[25] Lo, E.H., Dalkara, T. and Moskowitz, M.A. (2003) Mechanisms, Challenges and Opportunities in Stroke. Nature Reviews Neuroscience, 4, 399-415.

https://doi.org/10.1038/nrn1106

[26] Misra, S., Hascall, V.C., Markwald, R.R., O’Brien, P.E. and Ghatak, S. (2018) Inflammation and Cancer. In: Turksen, K., Ed., Wound Healing: Stem Cells Repair and Restorations, Basic and Clinical Aspects, Wiley, Hoboken.

[27] Awooda, H.A., Sharara, G.M., Soltani, N. and Saeed, A.M. (2014) Tumor Necrosis Factor- $\alpha$ and Nuclear Factor Kappa- $\beta$ Expression in Rats Following Transient Focal Cerebral Ischemia Reperfusion. International Journal of Clinical and Experimental Neurology, 2, 40-46.

[28] Dotan, Y., Lichtenberg, D. and Pinchuk, I. (2004) Lipid Peroxidation Cannot Be Used as a Universal Criterion of Oxidative Stress. Progress in Lipid Research, 43, 200-227.

[29] Adibhatla, R.M. and Hatcher, J.F. (2010) Lipid Oxidation and Peroxidation in CNS Health and Disease: From Molecular Mechanisms to Therapeutic Opportunities. Antioxidants \& Redox Signaling, 12, 125-169. https://doi.org/10.1089/ars.2009.2668

[30] Emerit, J., Edeas, M. and Bricaire, F. (2004) Neurodegenerative Diseases and Oxidative Stress. Biomedicine and Pharmacotherapy, 58, 39-46. https://doi.org/10.1016/j.biopha.2003.11.004

[31] Cherubini, A., Ruggiero, C., Polidori, M.C. and Mecocci, P. (2005) Potential Markers of Oxidative Stress in Stroke. Free Radical Biology and Medicine, 39, 841-852. https://doi.org/10.1016/j.freeradbiomed.2005.06.025

[32] Uchida, K. (2003) Histidine and Lysine as Targets of Oxidative Modification. Ami- 
no Acids, 25, 249-257. https://doi.org/10.1007/s00726-003-0015-y

[33] Liu, H., Uno, M., Kitazato, K.T., Suzue, A., Manabe, S., Yamasaki, H., et al. (2004) Peripheral Oxidative Biomarkers Constitute a Valuable Indicator of the Severity of Oxidative Brain Damage in Acute Cerebral Infarction. Brain Research, 1025, 43-50. https://doi.org/10.1016/j.brainres.2004.07.071

[34] Love, S., Barber, R. and Wilcock, G.K. (2000) Neuronal Death in Brain Infarcts in Man. Neuropathology and Applied Neurobiology, 26, 55-66. https://doi.org/10.1046/j.1365-2990.2000.00218.x

[35] Love, S. (2003) Apoptosis and Brain Ischaemia. Progress in Neuro-Psychopharmacology and Biological Psychiatry, 27, 267-282. https://doi.org/10.1016/S0278-5846(03)00022-8

[36] Valavanidis, A., Vlachogianni, T. and Fiotakis, C. (2009) 8-Hydroxy-2'-Deoxyguanosine (8-OHdG): A Critical Biomarker of Oxidative Stress and Carcinogenesis. Journal of Environmental Science and Health Part C Environmental Carcinogenesis \& Ecotoxicology Reviews, 27, 120-139. 